\title{
Maternal MTHFR C677T polymorphism and congenital heart defect risk in the Chinese Han population: a meta-analysis
}

\author{
K.H. Chen, L.L. Chen, W.G. Li, Y. Fang and G.Y. Huang \\ Department of Cardiology, Fujian Longyan Hospital, Longyan, Fujian, China \\ Corresponding author: K.H. Chen \\ E-mail: chen.kaihong@yahoo.cn
}

Genet. Mol. Res. 12 (4): 6212-6219 (2013)

Received November 12, 2013

Accepted April 26, 2013

Published December 4, 2013

DOI http://dx.doi.org/10.4238/2013.December.4.8

\begin{abstract}
Numerous studies have evaluated the association between the maternal C677T polymorphism in the methylenetetrahydrofolate reductase (MTHFR) gene and congenital heart defect (CHD) risk in the Chinese Han population. However, the specific association is still controversial. Six separate studies with 1089 subjects in the Chinese Han population on the relationship between the C677T polymorphism and CHDs were analyzed by meta-analysis, upon database search. The fixed-effect model or random-effect model was selected to calculate the pooled odds ratio (ORs) and its corresponding 95\% confidence interval $(95 \% \mathrm{CI})$ when appropriate. The Begg test was used to measure publication bias. Sensitivity analyses were performed to insure authenticity of the outcome. Meta-analysis of the results showed significant associations between the maternal $\mathrm{C} 677 \mathrm{~T}$ polymorphism and CHD risk ( $\mathrm{CC} v s \mathrm{TT}$ : $\mathrm{OR}=0.65,95 \% \mathrm{CI}=0.44-0.96)$. Limiting the analysis to the studies with controls in the Hardy-Weinberg equilibrium and the results indicate that the meta-analysis was statistically significant. Results of Begg's funnel plot showed that there was no publication bias (all $\mathrm{P}>0.05$ ). The present meta-analysis suggested
\end{abstract}


that the maternal C677T polymorphism is a risk factor for CHDs in the Chinese Han population.

Key words: Methylenetetrahydrofolate reductase; Gene polymorphism; Congenital heart defects; Meta-analysis

\section{INTRODUCTION}

Congenital heart disease (CHD) is a category of heart disease that includes abnormalities in cardiovascular structures that occur before birth, which, although vary throughout the world, are the most common developmental anomaly and the leading non-infectious cause of mortality in newborns (Garg et al., 2003).

The incidence of CHDs in underdeveloped countries is not known, and in the Western industrialized world has varied from a low value of about 3 to 5 per 1000 live births to about 12 per 1000 live births (Hoffman, 1995). To date, the cause of CHDs is unknown. However, there are some factors associated with an increased chance of having CHDs. These risk factors include: 1) taking certain medications or alcohol or drug abuse during pregnancy and 2) maternal viral infection, such as rubella (German measles) in the first trimester of pregnancy. In addition, genetic factors have been implicated in the pathogenesis of CHDs (Bruneau, 2008).

Evidence suggests that supplementation of folic acid reduces the risk of CHDs (Botto et al., 2003). In an Atlanta PCC study, use of periconceptional multivitamins containing folic acid was associated with a $24 \%$ reduction in the odds for congenital heart defects in general (Botto et al., 2000). The precise role of folate supplementation on cardiac morphogenesis remains unclear. Folic acid might have a role in the migration of the cardiac neural crest cells that contribute to the formation of the truncus arteriosus and its division into the aorta and pulmonary artery, thus probably affecting the conotruncal defects in particular (Tang et al., 2004; Van Beynum et al., 2006).

5,10-Methylenetetrahydrofolate reductase (MTHFR) is an important enzyme in homocysteine metabolism and catalyzes the reduction of 5,10-methylenetetrahydrofolate into 5-methyltetrahydrofolate, the predominant circulating form of folate. In the MTHFR enzyme, a common $\mathrm{C}$ to $\mathrm{T}$ substitution at position 677 (C677T) exists, resulting in a substitution of alanine to valine, causing impaired folate binding and reduced activity of the MTHFR enzyme. In recent years, several studies found an association between the maternal MTHFR C677T polymorphism and risk of CHDs. However, the published results have been inconsistent. Thus, to clarify this inconclusive relationship, we performed a meta-analysis of all available studies to demonstrate the association of the maternal C677T polymorphism with the susceptibility to CHDs in the Chinese Han population.

\section{MATERIAL AND METHODS}

\section{Literature search}

PubMed, Google scholar and China National Knowledge Infrastructure database searches were performed to retrieve papers linking the C677T polymorphism in the MTHFR 
gene and CHD risk, available on line by July 2012 without language restrictions. The following key words: "C677T", "methylenetetrahydrofolate reductase/MTHFR", "gene polymorphism", "congenital heart defects/CHDs", and "single nucleotide polymorphism" were used. In addition, a manual search of reference lists for original articles was performed. The reference lists of major textbooks, review articles, and of all the included articles identified by the search were then individually searched to find other potentially eligible studies.

\section{Inclusion and exclusion criteria}

Studies satisfying the following criteria were included: a) case-control studies that addressed CHD cases and healthy controls; $b$ ) studies that evaluated the association between the C677T polymorphism and CHD risk; c) all patients with clinically diagnosed CHDs; d) studies that included sufficient genotype data for extraction. Studies were excluded when: a) not case-control studies that evaluated the association between the C677T polymorphism and CHD risk; b) case reports, letters, reviews, and editorial articles; c) studies that were based on incomplete raw data or no usable data reported; d) duplicate data were contained in the studies; e) family-based design.

\section{Data extraction}

Two reviewers independently performed data extraction and then checked the results together. The following information was extracted from the included studies: first author, year of publication, area, number of cases and controls, genotype frequencies in cases and controls, and evidence of Hardy-Weinberg equilibrium (HWE) in controls (Table 1). For conflicting evaluations, an agreement was reached following a discussion.

\section{Statistical analysis}

The Fisher exact test was used to assess the HWE with the significance set at $\mathrm{P}<$ 0.05. The association between the C677T polymorphism and CHDs was compared using the odds ratio (OR) corresponding to a $95 \%$ confidence interval $(95 \% \mathrm{CI})$. The pooled ORs were performed for a co-dominant model (CC vs TT, CC vs TC), a dominant model (TT+CT $\left.v_{s} \mathrm{CC}\right)$, and a recessive model (CC+TC $\left.v s \mathrm{TT}\right)$. The $Q$ test and the $I^{2}$ test were performed to quantify the proportion of the total variation due to heterogeneity. $I^{2}$ ranges from 0 to $100 \%$. A value of $0 \%$ indicates no observed heterogeneity, and larger values reflect increasing heterogeneity, with $25 \%$ regarded as low, $50 \%$ as moderate, and $75 \%$ as severe heterogeneity. When the effects were assumed to be homogeneous ( $\left.\mathrm{P}>0.1, I^{2}<50 \%\right)$, the fixed-effect model was used in the absence of heterogeneity. Otherwise, the random-effect model was used. Sensitivity analysis was performed by removing the studies in the meta-analysis due to the genotype distribution in the control groups of the study deviating from HWE. The funnel plot asymmetry was assessed by the Begg test to estimate the potential publication bias (the significance was set at the $\mathrm{P}<0.05$ ). The statistical analyses were performed by the STATA 12.0 software (StataCorp., College Station, TX, USA). 


\section{RESULTS}

\section{Studies included in the meta-analysis}

The search strategy retrieved 146 potentially relevant studies. Based on the inclusion criteria, only 6 case-control studies (Liu et al., 2002; Yan et al., 2003; Zhu et al., 2006; Zhong et al., 2006; Li et al., 2002, 2007) with full-text were included in this meta-analysis and 140 studies were excluded. The flow chart of study selection is summarized in Figure 1. These 6 case-control studies included a total of 589 CHD cases and 500 healthy controls. All studies included were case-control studies, which evaluated the association between the maternal C677T polymorphism and susceptibility to CHDs. The published year of the included studies ranged from 2000 to 2012. The source of controls was based on healthy populations. The HWE test was performed on genotype distribution of the controls, and all of them were in agreement with HWE except for one study (Li, 2002). The study characteristics are presented in Table 1.

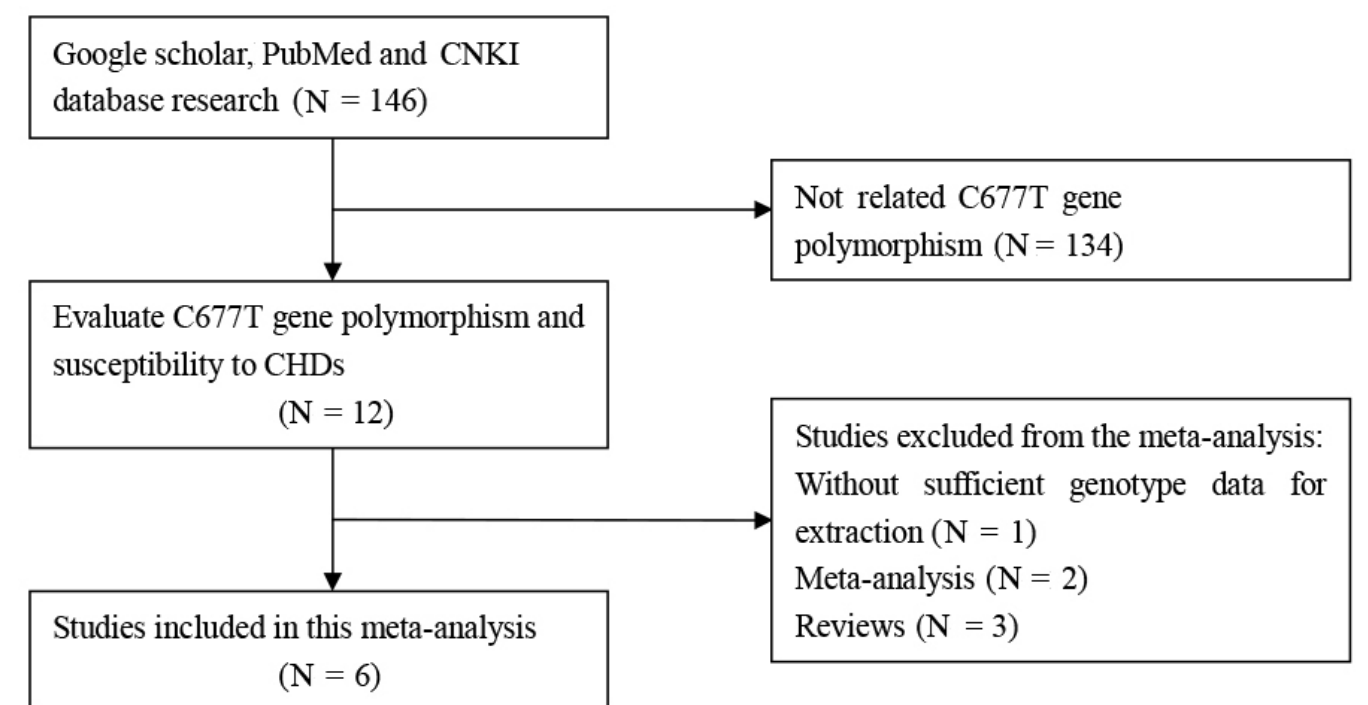

Figure 1. Flow diagram of study searching and selection process.

\begin{tabular}{|c|c|c|c|c|c|c|c|c|c|c|c|}
\hline \multirow[t]{2}{*}{ Study included } & \multirow[t]{2}{*}{ Year } & \multirow[t]{2}{*}{ Area } & \multirow[t]{2}{*}{ Cases } & \multirow[t]{2}{*}{ Controls } & \multicolumn{3}{|c|}{ Genotypes for cases } & \multicolumn{3}{|c|}{ Genotypes for controls } & \multirow[t]{2}{*}{ HWE test } \\
\hline & & & & & TT & $\mathrm{TC}$ & $\mathrm{CC}$ & TT & $\mathrm{CT}$ & $\mathrm{CC}$ & \\
\hline Li et al. & 2002 & Liaoning & 174 & 97 & 55 & 90 & 29 & 22 & 55 & 20 & 0.18 \\
\hline Liu et al. & 2002 & Beijing & 27 & 20 & 8 & 14 & 5 & 3 & 15 & 2 & 0.02 \\
\hline Yan et al. & 2003 & Liaoning & 187 & 102 & 61 & 94 & 32 & 25 & 57 & 20 & 0.22 \\
\hline Zhu et al. & 2006 & Beijing & 56 & 102 & 23 & 27 & 6 & 25 & 57 & 20 & 0.22 \\
\hline Zhong et al. & 2006 & Guangxi & 115 & 115 & 15 & 33 & 67 & 5 & 34 & 76 & 0.63 \\
\hline Li et al. & 2007 & Shandong & 104 & 208 & 46 & 42 & 16 & 39 & 114 & 55 & 0.14 \\
\hline
\end{tabular}




\section{Quantitative synthesis}

The evaluation of association between the maternal C677T polymorphism and the risk of CHDs is displayed in Table 2. The association between the maternal C677T polymorphism and CHDs in the Chinese Han population was significant under CC vs TT (OR $=0.65,95 \% \mathrm{CI}$ $=0.44$ to 0.96 ; Figures 2 and 3). Sensitivity analysis was performed by omission of one nonHWE study ( $\mathrm{Li}, 2002)$ and the result was not altered, indicating that the result of meta-analysis was statistically significant (Table 2). The funnel plot was used to assess the publication bias. There was no evidence of publication bias visually from the funnel plot (Table 2), which implied that the publication bias was low in the present meta-analysis.

Table 2. Summary ORs and $95 \% \mathrm{CI}$ of the studies included for meta-analysis.

\begin{tabular}{|c|c|c|c|c|c|c|c|c|c|}
\hline \multirow[t]{2}{*}{ Subgroup } & \multirow[t]{2}{*}{ Genetic model } & \multicolumn{2}{|c|}{ Sample size } & \multirow[t]{2}{*}{ Type of model } & \multicolumn{2}{|c|}{ Test of heterogeneity } & \multirow{2}{*}{$\begin{array}{l}\text { Test of association } \\
\text { OR }(95 \% \mathrm{CI})\end{array}$} & \multicolumn{2}{|c|}{ Test of publication bias } \\
\hline & & Case & Control & & $\mathrm{I}^{2}$ & $\mathrm{P}$ & & $\mathrm{z}$ & $\mathrm{P}$ \\
\hline \multirow[t]{4}{*}{ Overall } & $\mathrm{CC} v s \mathrm{TT}$ & 663 & 644 & Random & $67.1 \%$ & 0.01 & $0.48(0.25-0.93)$ & 0.34 & 0.73 \\
\hline & $\mathrm{CC}$ vs TC & & & Fixed & $43.7 \%$ & 0.11 & $1.03(0.77-1.37)$ & 0.34 & 0.73 \\
\hline & Dominant model & & & Fixed & $0.0 \%$ & 0.63 & $1.45(1.09-1.91)$ & 0.34 & 0.73 \\
\hline & Recessive model & & & Fixed & $18.1 \%$ & 0.30 & $0.46(0.35-0.61)$ & 0.34 & 0.73 \\
\hline \multirow{4}{*}{$\begin{array}{l}\text { Consistent } \\
\text { with HWE }\end{array}$} & $\mathrm{CC} v s \mathrm{TT}$ & 636 & 624 & Random & $73.1 \%$ & 0.01 & $0.45(0.22-0.93)$ & 0.00 & 1.00 \\
\hline & $\mathrm{CC}$ vs TC & & & Fixed & $48.3 \%$ & 0.10 & $1.00(0.75-1.34)$ & 0.00 & 1.00 \\
\hline & Dominant model & & & Fixed & $0.0 \%$ & 0.74 & $1.49(1.12-1.98)$ & 0.00 & 1.00 \\
\hline & Recessive model & & & Fixed & $34.3 \%$ & 0.19 & $0.46(0.35-0.61)$ & 0.00 & 1.00 \\
\hline
\end{tabular}

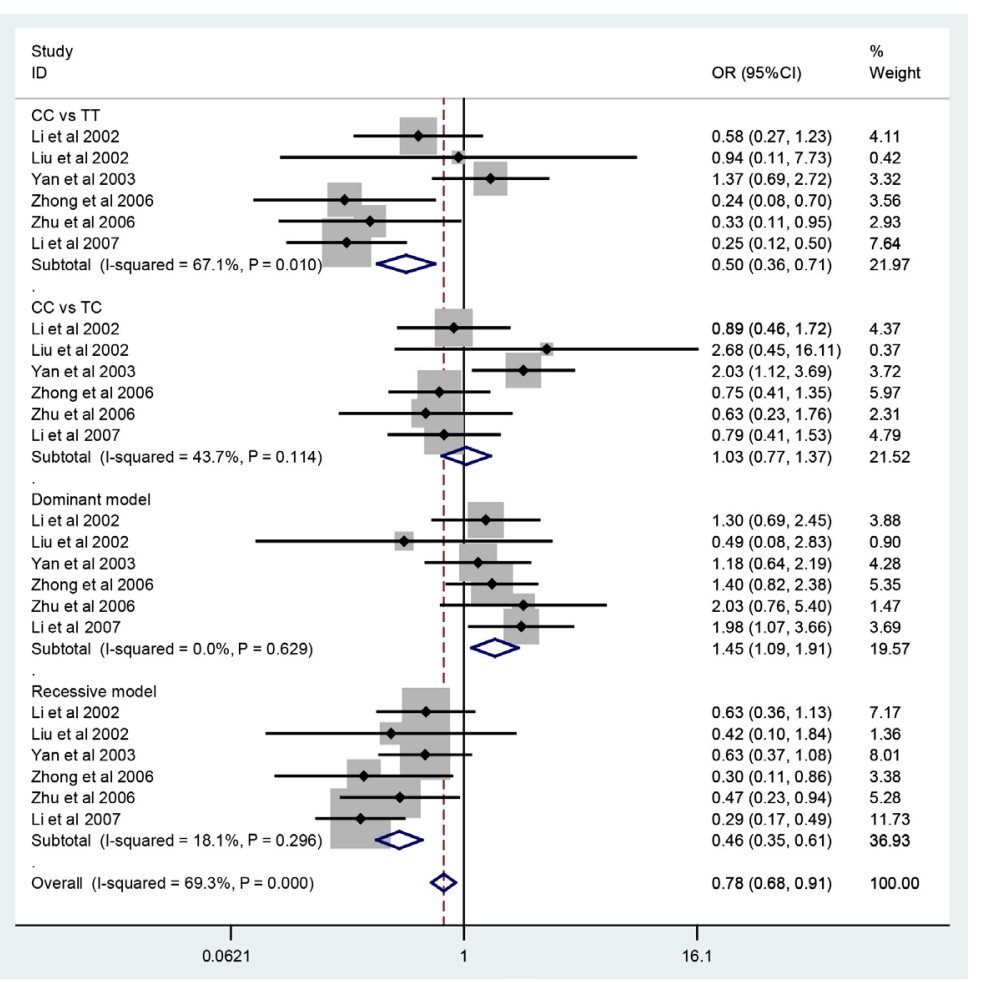

Figure 2. Association of the C677T polymorphism with the risk for CHDs in the Chinese Han population (CC vs TT). 


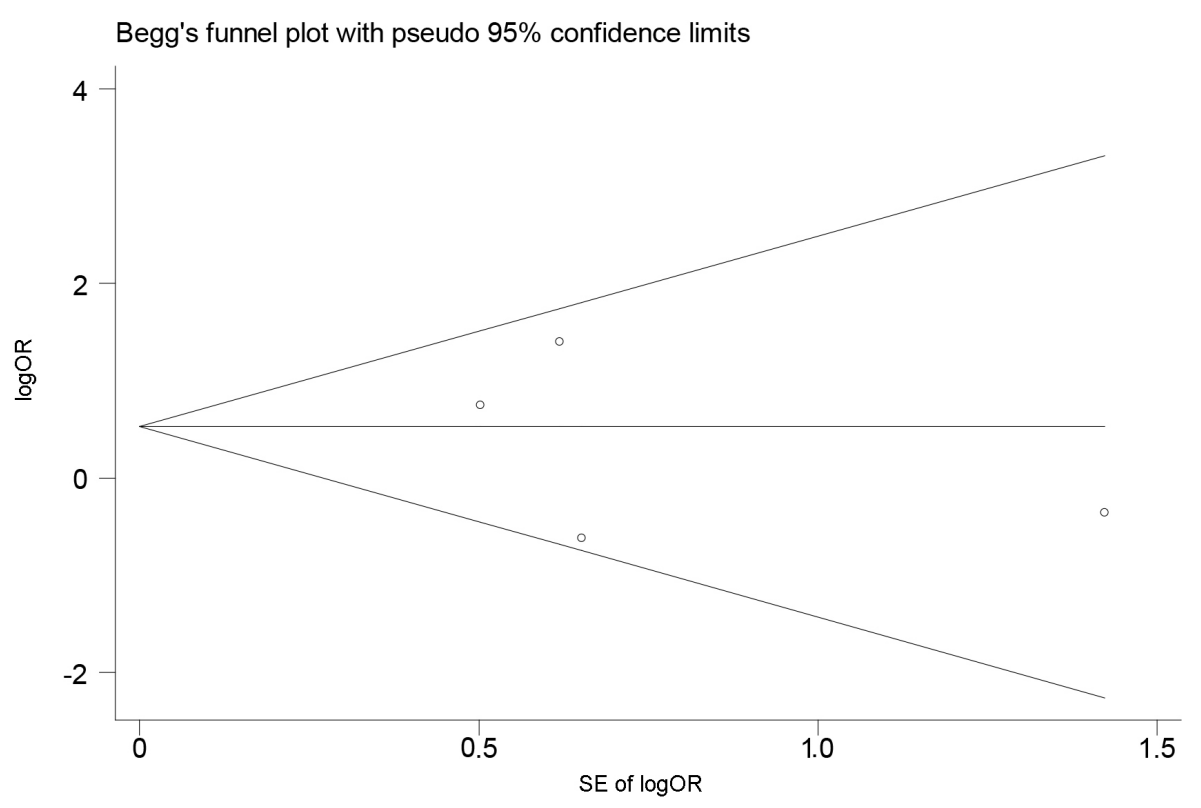

Figure 3. Funnel plot of the C677T polymorphism and susceptibility of CHDs in the Chinese Han population (CC vs TT).

\section{DISCUSSION}

As known, genetic polymorphisms altering the level of protein expressed would be anticipated to have a substantial influence on disease activity (Tahara et al., 2009). The relationship between the C677T polymorphism and CHD risk has been well studied, and the results are controversial. The aim of meta-analysis is to combine the same kind of studies to increase the sample size and statistical power, and thereby get a more authentic result. This is the first systematic study of the association between the C677T polymorphism and CHD risk in Han Chinese using the meta-analysis, and the results revealed that the maternal C677T polymorphism in the MTHFR gene were significantly associated with the susceptibility of CHDs (CC vs TT: OR $=0.65,95 \% \mathrm{CI}=0.44-0.96)$. We only selected the relatively homogeneous Han Chinese in our study to enhance statistical power and reduce genetic heterogeneity (Niu et al., 2009). To date, our study with an analysis of 1089 participants, provides the most comprehensive evaluation on the association between the maternal C677T variant and CHDs in Han Chinese. Further sensitivity analysis confirmed the significant association between the maternal C677T polymorphism and CHD risk among the Chinese population. No evidence showed publication bias in this meta-analysis for the C677T polymorphism. As the eligible study number was limited in the meta-analysis, these results still need further investigation.

However, previous meta-analysis mainly aiming at Europeans did not observe significant association between the maternal C677T polymorphism and CHDs (van Beynum et al., 2006). A comparable mechanism might be that maternal periconceptional use of folic acid and multivitamin supplements contain more folic acid in Europe than in China (Li et al., 2007; 
van Beynum et al., 2007); a higher dietary intake of folic acid has been shown to be effective in increasing folate concentration in both serum and red blood cells among women of childbearing age since the period of pregnancy (FDA, 1996). van Beynum et al. (2006) studied the association between the maternal MTHFR C677T polymorphism and periconceptional folate supplementation on the risk of CHDs in offspring, and mothers with the MTHFR 677TT genotype who did not use periconceptional folate supplements had a 2-fold increased risk of a CHD-affected child. The maternal MTHFR 677CT and TT genotypes were no genetic risk factors for CHD in women using folate supplements during the entire advised period (van Beynum et al., 2006). Using folic acid antagonists during pregnancy add the risk of CHDs (Sharfstein, 2001). Thus, we recommend that Chinese women increase their folic acid and multivitamin intake during pregnancy.

The mechanism of how the maternal C677T polymorphism relates to CHD risk is still unclear. A possible reason is that the homocysteine levels were shown to be increased with the 677TT genotype. About 10-12\% of Caucasians of Northern European descents carry this $677 \mathrm{TT}$ genotype, and they have $25 \%$ higher homocysteine levels than those with the $677 \mathrm{CC}$ genotype. The homocysteine methylation cycle may play a causal role in the development of CHDs, interfering with the developing heart by affecting neural crest cells (Jacques et al., 1996). This evidence suggests that the C677T polymorphism might play an important role in the development of CHDs.

There are still some limitations in this meta-analysis. Firstly, because of incomplete raw data or publication limitations, some relevant studies could not be included in our analysis. Secondly, the number of published studies was not sufficiently large for a comprehensive analysis, and some included studies of small size might not have had enough statistical power to explore the real association between the C677T polymorphism and susceptibility to CHDs. Finally, the genotype information stratified for the main confounding variables was not available in the original papers, such as age, gender, ethnicity, and exposures, which might cause serious confounding bias.

In conclusion, these results indicate significant association of the maternal C677T polymorphism with CHD susceptibility in the Chinese population. As long as folate fortification of food products is not applied in most countries, the benefits of periconceptional folate supplementation must be proclaimed with more strength. However, further studies with consideration of gene-gene and gene-environment interactions should be conducted to further investigate this association.

\section{Conflicts of interest}

The authors declare no conflict of interest.

\section{REFERENCES}

Botto LD, Mulinare J and Erickson JD (2000). Occurrence of congenital heartdefects in relation to maternal multivitamin use. Am. J. Epidemiol. 151: 878-884.

Botto LD, Mulinare J and Erickson JD (2003). Do multivitamin or folic acid supplements reduce the risk for congenital heart defects? Evidence and gaps. Am. J. Med. Genet. A 121A: 95-101.

Bruneau BG (2008). The developmental genetics of congenital heart disease. Nature 451: 943-948.

FDA (1996). Food Standards: amendment of standards of identity for enriched grain products to require addition of folic acid. Fed. Reg. 61: 8781-8797. 
Garg V, Kathiriya IS, Barnes R, Schluterman MK, et al. (2003). GATA4 mutations cause human congenital heart defects and reveal an interaction with TBX5. Nature 424: 443-447.

Hoffman JI (1995). Incidence of congenital heart disease: I. Postnatal incidence. Pediatr. Cardiol. 16: 103-113.

Jacques PF, Bostom AG, Williams RR, Ellison RC, et al. (1996). Relation between folate status, a common mutation in methylenetetrahydrofolate reductase, and plasma homocysteine concentrations. Circulation 93: 7-9.

Li Y (2002). Effect of parental MTHFR genetype on offspring congenital heart disease risk. J. Hyg. Res. 31: 52-55.

Li Z, Ren A, Zhang L, Liu J, et al. (2007). Periconceptional use of folic acid in Shanxi Province of northern China. Publ. Health Nutr. 10: 471-476.

Liu H, Li S and Ye HM (2002). Maternal homocysteine folic acid, MTHFR gene polymorphism and congenital heart defects in offspring. Chin. J. Perinat. Med. 5: 102-105.

Niu WQ, Qi Y, Zhang LT, Qi YX, et al. (2009). Endothelial nitric oxide synthase genetic variation and essential hypertension risk in Han Chinese: the Fangshan study. J. Hum. Hypertens. 23: 136-139.

Sharfstein JM (2001). Folic acid antagonists during pregnancy and risk of birth defects. N. Engl. J. Med. 344: 933-935.

Tahara T, Shibata T, Nakamura M, Yamashita H, et al. (2009). Effect of polymorphisms in the 3' untranslated region (3'UTR) of vascular endothelial growth factor gene on gastric cancer and peptic ulcer diseases in Japan. Mol. Carcinog. 48: 1030-1037.

Tang LS, Wlodarczyk BJ, Santillano DR, Miranda RC, et al. (2004). Developmental consequences of abnormal folate transport during murine heart morphogenesis. Birth Defects Res. A Clin. Mol. Teratol. 70: 449-458.

van Beynum IM, Kapusta L, den Heijer M, Vermeulen SH, et al. (2006). Maternal MTHFR 677C $>$ T is a risk factor for congenital heart defects: effect modification by periconceptional folate supplementation. Eur. Heart J. 27: 981-987.

van Beynum IM, den Heijer M, Blom HJ and Kapusta L (2007). The MTHFR 677C->T polymorphism and the risk of congenital heart defects: a literature review and meta-analysis. QJM 100: 743-753.

Yan LY, Li SQ and Zhao HJ (2003). Effect of 5,10-methylenetetrahydro-folate reductase genotypes in parents on the risk of congenital heart disease in offspring. Chin. J. Dis. Control. Prev. 7: 94-97.

Zhong QA, Qiu XQ and Zeng XY (2006). Association of congenital heart diseases with MTHFR gene and CBS gene. $J$. Guangxi Med. 28: 1140-1142.

Zhu WL, Li Y, Yan L, Dao J, et al. (2006). Maternal and offspring MTHFR gene C677T polymorphism as predictors of congenital atrial septal defect and patent ductus arteriosus. Mol. Hum. Reprod. 12: 51-54. 